\title{
El derecho a la verdad en las violaciones graves a Derechos Humanos. Caso San Fernando, México)
}

\section{Luisa Gabriela Morales Vega*}

Enviado: 23 de Junio

Dictaminado: 16 de Agosto

\section{Resumen}

Es deber de todo Estado democrático garantizar el Derecho a la Verdad, mismo que deriva de los órdenes jurídicos nacional e internacional. A pesar de su reciente determinación, este derecho es de suma importancia al permitir el conocimiento de eventos que por su naturaleza ofenden profundamente no sólo a los individuos, sino a la sociedad e incluso a la humanidad misma. En México La principal garantía legal de este derecho, la constituye la Ley General de Transparencia y Acceso a la Información Pública (antes la Ley General de Transparencia y Acceso a la Información Pública, vigente cuando tuvo verificativo el caso que se analiza) que prevé la publicidad de las averiguaciones previas relativas a violaciones graves de Derechos Humanos, puesto que dichas violaciones exceden el interés particular a la privacidad. Empero, la redacción lacónica del texto legal no permite identificar la autoridad o instancia facultada para calificar la gravedad de las violaciones cometidas, sin obviar el debate sobre si es posible hablar de grados de gravedad entre las violaciones de los derechos fundamentales. La ausencia de una atribución legal específica a determinada instancia o autoridad anula el principio de máxima publicidad al que está sujeta la información gubernamental y obstaculiza el ejercicio del derecho a la verdad que debe gozar la ciudadanía de todo estado democrático ante situaciones de violaciones graves a los derechos humanos, como lo es la masacre ocurrida en San Fernando, Tamaulipas en 2010. Palabras clave: Derecho a la verdad, Violaciones graves a derechos humanos, Transparencia, Masacre San Fernando.

\footnotetext{
* Universidad Autónoma de México.1gmoralesv@uaemex.mx
} 


\title{
The right to the truth in serious violations of Human Rights. Case San Fernando, Mexico
}

\begin{abstract}
It is the duty of every democratic State to guarantee the Right to Truth, which derives from national and international legal orders. In spite of its recent determination, this right is very important in allowing the knowledge of events that by their nature deeply offend not only individuals, but society and even humanity itself. In Mexico the main legal guarantee of this right is the General Law on Transparency and Access to Public Information (formerly the General Law on Transparency and Access to Public Information, in force when the case being analyzed was verified), which provides for the publicity of previous inquiries concerning serious violations of Human Rights, since such violations exceed the particular interest for privacy. However, the laconic wording of the legal text does not allow the identification of the authority or body authorized to rate the seriousness of the violations committed, without neglecting the debate on whether it is possible to speak of degrees of seriousness between violations of fundamental rights. The absence of a specific legal attribution to a particular authority or authority annuls the principle of maximum publicity to which government information is subject and obstructs the exercise of the right to the truth that citizenship of every democratic state should enjoy in situations of serious violations of Human rights, such as the massacre that occurred in San Fernando, Tamaulipas in 2010.
\end{abstract}

Key words: Right to True, gross violations of human rights, publicity, San Fernando massacre

\section{Sumario}

I. Introducción; II. El derecho a la verdad; III. Régimen nacional en materia de transparencia y acceso a la información pública; IV. La masacre de San Fernando, Tamaulipas como ejemplo de violación grave a Derechos Humanos cometida en agravio de personas migrantes; V. Obstáculos al ejercicio del derecho a la verdad y a la información en México; VI. Conclusiones; Bibliografía 


\section{Introducción}

Setenta y dos personas migrantes fueron ejecutadas en el mes de agosto de 2010 en el estado de Tamaulipas, México; esta masacre se sumó a una ya larga serie de actos violentos que se han realizado en contra de migrantes en su tránsito por el territorio nacional.

A decir de los sobrevivientes de este trágico evento, los asesinatos fueron producto de la negativa del grupo de migrantes a colaborar con grupos de la delincuencia organizada y además fueron del conocimiento de algunas autoridades; lo que aunado a los motivos de la matanza y el número de víctimas mortales genera que el hecho indudablemente se trate de una grave violación a los derechos humanos de las personas implicadas.

Esta circunstancia por sí sola es suficiente para evitar que la información relativa a la investigación de los hechos, $\mathrm{y}$ todas las actuaciones relacionadas a ella, sea considerada como reservada, al actualizarse la excepción contenida en el último párrafo del artículo 14 de la hoy abrogada Ley de Transparencia y Acceso a la Información Pública Gubernamental.

En consecuencia, la negación a hacer pública la información relativa a la masacre de San Fernando anula el Derecho de acceso a la información que tienen las víctimas y sus familiares y ello obstaculiza de manera importante el Derecho a la Verdad que ellos mismos poseen y que además es de relevancia para toda la sociedad; de ahí la importancia de revisar cómo ha actuado el Estado, con miras a dilucidar quién estaría facultado para calificar la gravedad de las violaciones a los derechos humanos y la importancia que esta calificación tiene respecto del Derecho a la Verdad

Así, en el primer apartado se ofrece una caracterización del Derecho a la Verdad; más adelante se hace referencia al régimen jurídico sobre transparencia y acceso a la información pública en México; después se presenta un apartado en donde se establece qué debe entenderse por violaciones graves a derechos humanos, haciendo referencia específicamente a la masacre de San Fernando y por último se argumenta en torno a la limitación impuesta al Derecho a la Verdad que asiste a la sociedad en su conjunto, la cual se considera desproporcionada, pues la misma deriva de una omisión legal. 


\section{EI derecho a la verdad}

El derecho a la verdad es un concepto jurídico que ha sido deducido a partir de diversas disposiciones internacionales, regionales y nacionales; ello es necesario debido a la inexistencia de una prescripción concreta y explícita sobre el mismo.

Este derecho hace referencia a una obligación concreta atribuible a todos los Estados, la de proporcionar información a las víctimas, sus familiares o la sociedad en su conjunto sobre las circunstancias en que se cometieron violaciones graves a los derechos humanos (Naqvi, 2006)

A nivel internacional encontramos una referencia muy importante al mismo, en el Protocolo adicional II a los Convenios de Ginebra, un cuerpo normativo fundamental del Derecho Internacional Humanitario; lo que de inmediato permite relacionar este tipo de derechos entre aquéllos que se actualizan en situaciones de disrupción del orden jurídico y político instaurado, es decir en situaciones de emergencia o de aquéllas que por su gravedad comprometen fuertemente la normalidad estatal.

Lo que el artículo 32 del Protocolo II adicional a los Convenios de Ginebra de 1949 establece es que cuando se aplique la Sección en la que se inserta, que es la de "personas desaparecidas y fallecidas", las actividades de las Altas Partes contratantes, de las Partes en conflicto y de las organizaciones humanitarias internacionales mencionadas en los Convenios y en el presente Protocolo deberán estar motivadas ante todo por el derecho que asiste a las familias de conocer la suerte de sus miembros" (Comité Internacional de la Cruz Roja, 1977)

De esta disposición internacional se advierte la dimensión individual del derecho a la verdad al establecer como su titular a la familia cuyo miembro está desparecido o ha fallecido; no obstante, la Organización de las Naciones Unidas (ONU), lo ha caracterizado en dos dimensiones, la individual y la colectiva, al reconocer que es un derecho esencial para las víctimas, pero también para la sociedad en su conjunto, al prever que el esclarecimiento de la verdad sobre las violaciones de los derechos humanos del pasado puede ayudar a prevenir los abusos de los derechos humanos en el futuro (Organización de las Naciones Unidas, 2015) 
Así las cosas, es dable percibir la importancia social de este derecho, pues su respeto se convierte en un mecanismo para garantizar la no repetición de las violaciones a derechos humanos; de ahí que la propia ONU, apoye a los Estados en misiones de constatación de los hechos, las comisiones de investigación y las comisiones de verdad con el fin de descubrir la verdad sobre las violaciones graves a los derechos humanos y las violaciones serias del Derecho Internacional Humanitario (Organización de las Naciones Unidas, 2015) Lo cual es relevante si se toma en cuenta que estas violaciones serias del Derecho Internacional Humanitario se actualizan a través de conductas sistemáticas que llegan a configurar los crímenes internacionales previstos en el Estatuto de Roma.

Ahora, a nivel regional la disposición internacional de la que se deduce el Derecho a la Verdad, la encontramos en el artículo 13 de la Convención Americana sobre Derechos Humanos o Pacto de San José, mismo que contempla la Libertad de Pensamiento y de Expresión. La referencia al derecho a la verdad, se extrae del primer párrafo en donde textualmente se establece que Toda persona tiene derecho a la libertad de pensamiento y de expresión, este es un derecho complejo pues comprende la libertad de buscar, recibir y difundir informaciones e ideas de toda índole (Organización de Estados Americanos, 1969).

El texto del referido artículo 13, establece el vínculo entre el derecho a la verdad y el acceso a la información; podría parecer obvio dicho vínculo pero es importante explicitarlo pues como ya se ha dicho, no encontramos una mención textual, específica e individual del derecho a la verdad; no obstante, esa verdad únicamente es asequible cuando se garantiza la libertad de allegarse de información de cualquier índole y que este acceso sea no sólo respetado sino otorgado o garantizado por los Estados parte del Pacto de San José.

En ese sentido, el Sistema Interamericano de Derechos Humanos ha establecido que el derecho a conocer la verdad implica una obligación para el Estado respecto no sólo de las víctimas sino de la sociedad y es una consecuencia de las obligaciones y deberes que los Estados tienen por ser parte del Pacto de San José. (Comisión Interamericana de Derechos Humanos, 1999); en el mismo Informe, la Comisión reiteró que es un derecho de carácter colectivo que permite a la sociedad tener acceso a información esencial para el desarrollo de los sistemas democráticos y un derecho particular para los familiares de las 
víctimas, que permite una forma de reparación (Comisión Interamericana de Derechos Humanos, 1999)

Por su parte, la Corte Interamericana, ha incrementado la importancia de este derecho al proveer, dentro de casos relativos a desapariciones forzadas, que la ausencia de una investigación seria que esclarezca los hechos en situaciones de violación grave a los derechos de las personas, resultarían de alguna manera auxiliados por el poder público, lo que comprometería la responsabilidad internacional del Estado (Velásquez Rodríguez vs. Honduras, 1988)

Debido a que el derecho a la verdad ha sido esgrimido por la Corte Interamericana ante situaciones de desaparición forzada de personas, ejecuciones sumarias y masacres, existen varias sentencias dictadas contra diversos Estados en la región, que han abordado el tema; sin embargo, todas ellas coinciden en la doble dimensión de este derecho y la importancia del mismo en una sociedad democrática organizada constitucionalmente.

Estas referencias nos permiten comprender los alcances del derecho a la verdad, mismo que no se agota con una satisfacción al particular consistente en conocer lo que le ha ocurrido como víctima o familiar de la víctima y a partir de ella acceder a mecanismos de reparación; sino que también posee una dimensión social en el sentido de ser en sí mismo una garantía de no repetición al imponerse sobre la impunidad o la indolencia de la autoridad, además de que todas las posiciones que se han comentado, han derivado de asuntos relacionados a violaciones graves de Derechos Humanos, lo que genera que sea de interés general $\mathrm{y}$, adicionalmente, se trata de una obligación que impone el ser parte del Sistema Interamericano de Derechos Humanos y en el caso de México, dicha obligación es de primer orden al amparo del artículo $1^{\circ}$. de la Constitución Política de los Estados Unidos Mexicanos.

Para efectos de este artículo, es suficiente con la caracterización presentada del derecho a la verdad; no soslayamos la dificultad no sólo jurídica, sino filosófica de determinar a la verdad, como categoría de muchas corrientes del pensamiento, además de ser uno de los principales interrogantes de la humanidad. Pero para nuestros modestos fines, la verdad se presenta aquí únicamente como el derecho en relación al conocimiento que se debe tener de los actos que constituyen violaciones graves a los Derechos Humanos, es decir, su finalidad es práctica, por 
ello únicamente nos atenemos a conceptualizaciones también prácticas derivadas de la actuación de instituciones relacionadas con la impartición de justicia.

En el siguiente apartado se presenta el marco jurídico del Derecho de Acceso a la información.

\section{Régimen nacional en materia de transparencia y acceso a la información pública}

El artículo $6^{\circ}$. De la Constitución Política de los Estados Unidos Mexicanos, reconoce en su segundo párrafo que toda persona tiene derecho al libre acceso a la información plural y oportuna, asi como a buscar, recibir y difundir información e ideas de toda índole por cualquier medio de expresión, en este enunciado se basa el derecho de acceso a la información en México y además, el propio artículo en párrafos subsiguientes establece la obligación del Estado en materia de transparencia.

Dicha obligación se caracteriza en este artículo constitucional de la siguiente manera:

1. Tratándose de información pública, cualquier persona tiene el derecho de acceder a ella.

2. No es necesario acreditar interés alguno o justificar la utilización de la información solicitada

3. Será motivo de legislación secundaria establecer los mecanismos para acceder a la información.

4. Los sujetos obligados a brindar la información deberán preservar sus documentos en archivos administrativos actualizados

5. Tratándose de información relativa al ejercicio de recursos públicos, los sujetos obligados la publicarán por los medios electrónicos disponibles.

6. Se prevé la existencia de un organismo que será responsable de garantizar este derecho; dicho organismo será autónomo, especializado, imparcial, colegiado, con personalidad jurídica y patrimonio propio, con plena autonomía técnica, de gestión, capacidad para decidir sobre el ejercicio 
de su presupuesto y su organización interna. Este organismo es el Instituto Nacional de Acceso a la Información y Protección de Datos (INAI), cuyo antecesor el Instituto Federal de Acceso a la Información y Protección de Datos (IFAI) estaba en funciones en la época en que sucedió la matanza de San Fernando.

7. Dicho organismo se regirá por su respectiva ley bajo los principios de certeza, legalidad, independencia, eficacia, objetividad, profesionalismo, transparencia y máxima publicidad.

8. El organismo garante es competente para conocer de los asuntos relacionados con el acceso a la información pública y la protección de datos personales de cualquier autoridad, entidad, órgano u organismo que forme parte de alguno de los Poderes Legislativo, Ejecutivo y Judicial, órganos autónomos, partidos políticos, fideicomisos y fondos públicos, así como de cualquier persona física, moral o sindicatos que reciba y ejerza recursos públicos o realice actos de autoridad en el ámbito federal; con excepción de aquellos asuntos jurisdiccionales que correspondan a la Suprema Corte de Justicia de la Nación, en cuyo caso resolverá un comité integrado por tres ministros.

9. También conocerá de los recursos que interpongan los particulares respecto de las resoluciones de los organismos autónomos especializados de las entidades federativas que determinen la reserva, confidencialidad, inexistencia o negativa de la información, en los términos que establezca la ley.

Al tratarse de una libertad fundamental, no es necesario argumentar a favor de la importancia del derecho de acceso a la información y su estrecha correlación con la libertad de expresión; no obstante, de los elementos que contiene el artículo $6^{\circ}$. Constitucional y que fueron relatados en los párrafos precedentes, es necesario aclarar en qué consiste el principio de "máxima publicidad" puesto que el mismo no es definido ni en la constitución ni en las leyes reglamentarias que han derivado del mismo.

El principio de máxima publicidad establece la más amplia aplicación posible del derecho de acceso a la información que esté en posesión, custodia 
o control de la autoridad pública (Organización de Estados Americanos, 2010). Este principio implica también que toda información en posesión de cualquier autoridad es pública y sólo podrá ser reservada temporalmente por razones de interés público y seguridad nacional en los términos que fijen las leyes (FUNDAR Centro de Análisis e Investigación, 2015)

Por su parte, la Ley General de Transparencia y Acceso a la información Pública vigente, se refiere en el artículo 8, fracción VI expresamente a este principio, como un principio rector de los organismos garantes en materia de transparencia y textualmente dice: toda la información en posesión de los sujetos obligados será pública, completa, oportuna y accesible, sujeta a un claro régimen de excepciones que deberán estar definidas y ser además legítimas y estrictamente necesarias en una sociedad democrática.

Bajo esa óptica el principio de máxima publicidad pretende otorgar el acceso más amplio posible a la información pública al restringir su reserva únicamente por razones específicas y previstas en la ley. Es decir la reserva de la información pública debe ser decretarse de forma temporal y además esta reserva debe ser legalmente permitida.

La Ley de Transparencia y Acceso a la Información Pública Gubernamental vigente en 2010, cuando ocurrió la masacre de San Fernando, establecía en el artículo 14, los supuestos en que la información debía ser considerada como reservada, entre los cuales se encontraba el de las averiguaciones previas (fr. III); empero, el propio artículo en el último párrafo textualmente decía No podrá invocarse el carácter de reservado cuando se trate de la investigación de violaciones graves de derechos fundamentales o delitos de lesa humanidad.

Es decir, en virtud del principio de máxima publicidad absolutamente toda la información en posesión de las autoridades es pública y se debe garantizar el acceso a ella; ahora, el artículo 14 de la referida ley establecía excepciones a ese principio al señalar supuestos que a pesar de tratarse de información pública podría ser tratada como reservada y por ende, restringir o denegar el acceso a la misma; así, el último párrafo del artículo establecía a su vez una excepción a las excepciones, al disponer que si la información es relativa a violaciones graves a derechos fundamentales o delitos de lesa humanidad entonces no podría calificarse de reservada. 
Por su parte y a fin de constatar las similitudes con la ley vigente en la actualidad, la Ley de Transparencia y Acceso a la Información Pública, publicada el 9 de mayo de 2016, coincide en lo referente con su antecesora, de acuerdo a los artículos 3, 8 y 110 fracción XII de la misma. Los que señalan respectivamente que:

Toda la información generada, obtenida, adquirida, transformada o en posesión de los sujetos obligados en el ámbito federal, a que se refiere la Ley General de Transparencia y Acceso a la Información Pública y esta Ley, es pública, accesible a cualquier persona y sólo podrá ser clasificada excepcionalmente como reservada de forma temporal por razones de interés público y seguridad nacional o bien, como confidencial;

No podrá clasificarse como reservada aquella información que esté relacionada con violaciones graves a derechos humanos o delitos de lesa humanidad, de conformidad con el derecho nacional o los tratados internacionales de los que el Estado mexicano sea parte., y,

Conforme a lo dispuesto por el artículo 113 de la Ley General, como información reservada podrá clasificarse aquella cuya publicación Se encuentre contenida dentro de las investigaciones de hechos que la ley señale como delitos y se tramiten ante el Ministerio Público

Por su parte la Ley General de Transparencia y Acceso a la Información Pública vigente, estatuye en el artículo 4, segundo párrafo y 5, primer párrafo con referencia a la reserva de información lo siguiente:

Toda la información generada, obtenida, adquirida, transformada o en posesión de los sujetos obligados es pública y accesible a cualquier persona en los términos y condiciones que se establezcan en la presente Ley, en los tratados internacionales de los que el Estado mexicano sea parte, la Ley Federal, las leyes de las Entidades Federativas y la normatividad aplicable en sus respectivas competencias; sólo podrá ser clasificada excepcionalmente como reservada temporalmente por razones de interés público y seguridad nacional, en los términos dispuestos por esta Ley. 
No podrá clasificarse como reservada aquella información que esté relacionada con violaciones graves a derechos humanos o delitos de lesa humanidad, de conformidad con el derecho nacional o los tratados internacionales de los que el Estado mexicano sea parte.

Con las referencias legales aquí comentadas, es indudable que toda la información en posesión o control de la autoridad que se relacione con violaciones graves a derechos humanos debe hacerse pública y por consiguiente, difundirse por los medios establecidos en la propia ley, como garantía del Derecho a la Verdad.

La dificultad que se presenta en el caso de la masacre de San Fernando y que persiste, aun con la expedición de las leyes actuales, es la necesidad de dilucidar qué autoridad es la competente de calificar de graves a las violaciones de Derechos Humanos, bajo qué criterios y en qué momento. En los siguientes apartados este documento aporta algunos elementos que podrían superar dicha dificultad cuya verificación anula o entorpece el ejercicio del Derecho a la Verdad, mediante la violación del derecho de acceso a la información.

\section{La masacre de San Fernando, Tamaulipas como ejemplo de violación grave a Derechos Humanos cometida en agravio de las personas migrantes.}

Para comenzar este apartado es necesario considerar las acepciones que le han sido atribuidas a la frase violación grave a Derechos Humanos; para ello tenemos que desde el ámbito internacional, se ha denominado a determinado tipo de eventos o situaciones que vulneran derechos humanos como manifiestas, flagrantes, serias o graves.

Estos calificativos si bien son diversos entre sí, coinciden en que son atribuidas a eventos que atentan contra normas inderogables de derechos humanos, es decir normas imperativas cuyo cumplimiento no es negociable y mucho menos excusable por parte de los sujetos del Derecho Internacional. Este 
tipo de normas se refieren a un grupo de disposiciones que conforman un núcleo irreductible y obligatorio conocido como jus cogens.

Las normas a que nos referimos incluyen la prohibición de:

₹ la tortura y de cualquier trato cruel, inhumano o degradante;

$\approx$ la privación arbitraria de la vida;

$\approx$ la toma de rehenes;

$\approx$ la desaparición forzada;

$\approx \mathrm{y}$ los castigos colectivos;

Como se ve, se refiere a la protección de los derechos que por su importancia es obligación general respetarlos y protegerlos; también pueden conformar este núcleo el derecho de acceso a la justicia, el principio de igualdad y no discriminación (Cancado Trinidade, 2015)

Esta caracterización inicial sirve para deducir que una violación grave o seria es aquélla que atenta a los derechos considerados indiscutiblemente como primordiales, la vida, la integridad personal y la libertad.

En México, el Reglamento de la Comisión de Derechos Humanos del Distrito Federal considera como violaciones graves a los actos u omisiones que impliquen ataques al derecho a la vida, a la integridad física o psíquica de las personas, a la libertad, a la seguridad, así como aquellas que puedan afectar una colectividad o grupo de individuos (artículo 128)

Esta acepción como se ve, coincide con la deducida del derecho internacional pero se aprecia la dificultad de distinguir entre una violación grave o no, dicho de otro modo, admite la interrogante sobre si es posible tratándose de atentados a estos derechos calificar de grave o no grave, pues habrá algún atentado a la vida que no sea grave, o podrá existir una restricción arbitraria de la libertad que por dirigirse únicamente hacia un individuo, o por realizarse quizá en un estado de sitio pueda ser no grave.

A pesar de la apariencia retórica de las interrogantes del párrafo anterior, lo cierto es que es necesario intentar responderlas seriamente. Se podría sostener la opinión de que cualquier hecho que ataque la vida de una persona es una violación 
grave o a contrario sensu, no hay ataques contra la vida que sean no graves.

A fin de intentar resolver estas interrogantes, recurrimos a la tesis aislada pronunciada por la Primera Sala de la Suprema Corte de Justicia de la Nación en Febrero de 2012, que precisamente intenta establecer los criterios utilizables para calificar de grave una violación a derechos humanos con la finalidad de saber su repercusión sobre el derecho de acceso a la información, que es el tema que nos ocupa, y cuyo rubro y texto dicen:

VIOLACIONES GRAVES A DERECHOS HUMANOS. SU CONCEPTO PARA EFECTOS DEL DERECHO DE ACCESO A LA INFORMACIÓN DE LA AVERIGUACIÓN PREVIA QUE LAS INVESTIGA. De conformidad con el artículo 14 de la Ley Federal de Transparencia y Acceso a la Información Pública Gubernamental, no puede alegarse el carácter de reservado cuando la averiguación previa investigue hechos constitutivos de graves violaciones a derechos humanos o delitos de lesa humanidad. A fin de que el intérprete determine si un caso concreto se ubica en el supuesto de excepción relativo a las violaciones graves a derechos humanos y deba dar acceso a la averiguación previa correspondiente, es necesario que atienda a los lineamientos sentados por la Suprema Corte de Justicia de la Nación y por la Corte Interamericana de Derechos Humanos en esta materia. Siguiendo los lineamientos establecidos por este alto tribunal, para determinar que una violación a derechos humanos es "grave" se requiere comprobar la trascendencia social de las violaciones, lo cual se podrá determinar a través de criterios cuantitativos o cualitativos. El criterio cuantitativo determina la gravedad de las violaciones demostrando que tienen una trascendencia social en función de aspectos medibles o cuantificables, tales como el número, la intensidad, la amplitud, la generalidad, la frecuencia o su prolongación en el tiempo, así como, evidentemente, la combinación de varios de estos aspectos. Es lógico que el criterio anterior no haya podido aplicarse a todos los casos, razón por la cual esta Suprema Corte también ha entendido que en algunos supuestos la trascendencia social de las violaciones se puede demostrar mediante un criterio cualitativo, determinando si éstas presentan alguna característica o cualidad que les dé una dimensión especifica. En lo que respecta a la jurisprudencia de la Corte Interamericana de Derechos Humanos, ese tribunal ha determinado que la "gravedad" radica, esencialmente, en que se presenten las siguientes características: multiplicidad de violaciones comprendidas dentro del fenómeno delictivo; especial magnitud de las violaciones en relación a la naturaleza de los derechos afectados; 
y una participación importante del Estado, al ser los actos cometidos por agentes estatales o con la aquiescencia, tolerancia o apoyo del Estado. (Violaciones graves a derechos humanos. Su concepto para efectos del derecho de acceso ala información de la averiguación previa que las investiga, 2012)

La tesis transcrita, si bien es aislada, aporta algunos elementos para identificar la gravedad de las violaciones, pues dice en primer término que se requiere comprobar la trascendencia social de las violaciones, lo que vincula esta cualidad con el derecho a la verdad.

Después dice que existen criterios de tipo cuantitativo y cualitativo. El criterio cuantitativo se vale de verificar la existencia o concurrencia de aspectos medibles tales como número, intensidad o frecuencia de las violaciones; mientras que el criterio cualitativo se refiere a la característica o cualidad que les dé una dimensión específica como la multiplicidad de las violaciones, la especial magnitud, la participación importante del Estado, sea por realización, apoyo, tolerancia o aquiescencia.

De lo anterior se concluye que las violaciones que puedan ser calificadas como graves deben atentar contra derechos tutelados por normas inderogables que, si bien no es posible establecer una jerarquía entre derechos, lo cierto es que existen ciertos bienes jurídicos tutelados de mayor importancia pues constituyen la base indiscutible de otros, como lo son la vida, la libertad y la seguridad; además podemos agregar que, siguiendo el criterio de la SCJN, que en este tipo de violaciones deben verificarse ciertas condiciones cuantificables o no, pero que generan una mayor vulnerabilidad a las víctimas y sus familiares, pero que adicionalmente, son de tal trascendencia que es de interés de toda la sociedad su prevención, investigación, esclarecimiento y eventual sanción o reparación.

Los homicidios perpetrados en 2010 en contra de 72 personas migrantes en el municipio de San Fernando, Tamaulipas puede ser calificada como violación grave a Derechos Humanos; si bien es cierto, no es el único evento violento acontecido en contra de este grupo vulnerable, sino que por el contrario, en los últimos años se ha documentado incesantemente una intensa actividad criminal y violenta en contra de los migrantes, en su mayoría centroamericanos en tránsito 
por México. ${ }^{1}$ Del mismo modo, constituye un hecho notorio, las denuncias reiteradas del sacerdote Alejandro Solalinde y los encabezados de un sinfín de notas y editoriales publicadas en periódicos impresos y electrónicos.

La masacre sucedió el 22 de agosto de 2010, luego que la noche anterior un grupo de 74 personas migrantes que viajaba en dos camiones rumbo a la frontera norte del país fue interceptado por un grupo armado, los hechos se dieron a conocer el 25 de agosto de 2010 a través de una publicación electrónica de E1 Universal, en la que se leía que un hombre se había presentado en un puesto de control carretero de la Secretaría de Marina en busca de asistencia médica para atender una herida de bala producida por un grupo delictivo que lo interceptó junto a otros migrantes a quienes asesinaron por negarse a trabajar con ellos, según el dicho de dos sobrevivientes de la matanza identificados como V73 y V74. (Comisión Nacional de Derechos Humanos, 2013)

La recomendación emitida por la $\mathrm{CNDH}$, expresa literalmente en el párrafo 106 que debido a que los hechos revisten relevancia de carácter nacional, el 29 de agosto de 2010, la Procuraduría General de la República resolvió ejercer la facultad de atracción de la Averiguación Previa iniciada por la Procuraduría General de Justicia del Estado de Tamaulipas. (Comisión Nacional de Derechos Humanos, 2013) Ello sin mencionar afirmaciones más bien especulativas que trascendieron en el sentido de que las propias autoridades entregaron los migrantes al grupo delictivo.

La atracción de la Averiguación Previa iniciada por la procuraduría local que ejerció la Procuraduría General de la República por tratarse de hechos de relevancia nacional, constituye un elemento sólido para calificar de grave el evento, mismo que aunado a la cantidad de víctimas mortales del mismo y el contexto de violencia en el que se desarrolló permite de manera clara establecer que se estaba ante una violación grave de derechos humanos.

A más de lo anterior, meses después de la masacre, continuaron los crímenes en contra de migrante pue en abril de 2011, se descubrieron 43 restos humanos

\footnotetext{
1 Los principales delitos cometidos en agravio de migrantes son el secuestro y la extorsión, según informes emitidos por la Comisión Nacional de Derechos Humanos, Comisión Interamericana de Derechos Humanos, Consejo Nacional para Prevenir la Discriminación, Amnistía Internacional entre otros; a estos eventos nos hemos referido ampliamente en otros artículos.
} 
en una fosa clandestina y a partir de ella se registró el descubrimiento de 47 fosas más y 193 restos humanos en total. (FUNDAR, 2015)

Si después se toma en cuenta que durante la investigación de los hechos materia de la recomendación referida se presentaron obstáculos y dilación en la colaboración por parte de la Procuraduría General de la República, en el sentido de haberse impedido a esta comisión (la CNDH) el acceso inmediato a la información que obra en su poder, indispensable para conocer respecto de la verdad histórica, la identidad de las víctimas y constatar que la autoridad ministerial observaba los preceptos del orden jurídico (Comisión Nacional de Derechos Humanos, 2013) entonces es posible afirmar el carácter de grave de la situación y la complicación en su investigación, a lo que se suma la opacidad en la que se ha desarrollado la misma, pues como se verá en el siguiente apartado no fue sino hasta mayo de 2017 que la Suprema Corte de Justicia de la Nación se pronunció sobre la instancia que posee facultades para calificar de graves las violaciones y con ello poder difundir la información respecto de las mismas y garantizar el derecho a la verdad.

\section{Obstáculos al ejercicio del derecho a la verdad y a la información en México}

Tratándose de violaciones graves a derechos humanos, como ya quedó asentado, no se actualiza la excepción al principio de Máxima Publicidad que preveía el artículo 14 de la hoy abrogada Ley General de Transparencia y Acceso a la Información Pública Gubernamental.

$\mathrm{Si}$, como se argumenta en el apartado anterior, los hechos sucedidos el $22 \mathrm{de}$ agosto de 2010 por el que fueron ejecutadas arbitrariamente 72 personas migrantes en el territorio nacional, por sus características y el contexto en el que sucedieron, constituyen una violación grave a derechos humanos, entonces la información relativa no es susceptible de reservarse. De ahí que surja la interrogante sobre las razones que han prevalecido para su reserva, hasta siete años después.

En 2015 un grupo de organizaciones civiles defensoras de Derechos 
Humanos solicitó a la SCJN que se pronunciara en el sentido de que asiste al Instituto Nacional de Acceso a la Información (INAI) la facultad de determinar cuándo es grave una violación y en consecuencia publicar la información (FUNDAR, 2015). La solicitud fue presentada a través de la figura amico curiae a los amparos en revisión números 661/2014 y 453/2015 radicados en la Primera Sala.

Esto después de que el $1^{\circ}$. de octubre de 2014 un tribunal federal confirmara la reserva de las averiguaciones previas relativas con fundamento en el artículo 14, fracción III tantas veces citado; no obstante contra dicha resolución se promovió juicio de amparo al que le correspondió el número 1371/2013 en el que el Juez $8^{\circ}$. de Distrito en Materia Administrativa en el Distrito Federal, determinó que el IFAI sí tiene facultades para pronunciarse prima facie sobre violaciones graves de derechos humanos únicamente para efectos de acceso y que la averiguación previa sobre la matanza de San Fernando sí envuelve investigaciones prima facie sobre violaciones graves a derechos Humanos (Instituto Federal de Acceso a la Información y Protección de Datos, 2015)

En dicho fallo, según el informe que se revisa, el Juez caracterizó a los hechos de acuerdo a criterios jurisprudenciales nacionales e internacionales y limitó el reconocimiento de gravedad del IFAI a su materia sustantiva que es la referente al acceso a la información, lo que no implica prejuzgar respecto de la responsabilidad penal de los sujetos implicados.

Ahora bien, no existe disposición alguna en las legislaciones en materia de transparencia de las que sea posible deducir la facultad del INAI para realizar la calificación necesaria. Por otro lado, la naturaleza y principal encomienda de este Instituto es velar por el efectivo goce del derecho de acceso a la información, torna aceptables los argumentos del Juez $8^{\circ}$. de Distrito. Por ello es que -suponemosexistió un debate al interior del INAI en el cual la comisionada ponente Jaqueline Peschard se pronunció a favor de la desclasificación de la información, pues la gravedad de los hechos lo ameritaba aun cuando ninguna autoridad en materia de Derechos Humanos se hubiese pronunciado en ese sentido; empero, el resto de los comisionados se decantaron por la clasificación.

En síntesis, existen voces que consideran que es el propio instituto el facultado para pronunciarse sobre la gravedad de las violaciones y así lograr 
que se actualice la excepción prevista en el último párrafo del artículo 14 de la Ley General de Transparencia y Acceso a la Información Pública Gubernamental aplicable al caso, pero también existe la resistencia a ella bajo el argumento de que debe ser realizada por una autoridad en materia de Derechos Humanos.

Razonamiento que encontramos atinado, puesto que la Constitución Política de los Estados Unidos Mexicanos establece atribuciones precisas a la Comisión Nacional de Derechos Humanos; esto es, a partir de la reforma de 10 de junio de 2011, se traslada a la CNDH la facultad de investigar sobre violaciones graves a Derechos Humanos que anterior a la reforma le correspondía a la Suprema Corte de Justicia de la Nación.

La facultad de investigación, la prevé el artículo 102 constitucional en el último párrafo, y si bien no es una facultad obligatoria, pues el texto constitucional dice textualmente que podrá investigar, lo cierto es que lo ha venido haciendo desde el 2012, en los casos relativos a la masacre de Tanhuato, Michoacán; Apatzingan, Michoacán, Tlatlaya, México, Ocoyucan, Puebla y Chilpancingo, Guerrero.

Por otro lado, el mismo párrafo dispone que la investigación la realizará la CNDH a solicitud del Ejecutivo Federal, de alguna de las Cámaras del congreso de la Unión, de los Gobernadores o legislatura de los estados y cuando así lo juzgue conveniente.

Este último supuesto de procedencia de la investigación me parece útil a sostener que es la CNDH quien realmente está facultada para realizar la calificación de gravedad a que se refiere este trabajo; la razón es que si la función investigadora la desplegará cuando lo juzgue conveniente y esta función versa únicamente sobre violaciones graves a derechos humanos, tenemos que este párrafo constitucional está reconociendo a la CNDH la capacidad de juzgar cuándo sí y cuándo no se trata de una violación grave, pues deja a su arbitrio el investigar, pero siempre sobre violaciones graves.

En otras palabras, el constituyente permanente, estimó que debido a la especialización de la CNDH en la materia, es capaz de identificar violaciones graves. Lo que coincide con el comunicado de prensa emitido el 31 de mayo de 2017 por la SCJN que establece que la CNDH se encuentra plenamente posibilitada de hacer esa calificación atendiendo al cúmulo de facultades que le 
fueron otorgadas a nivel constitucional y legal

Hasta aquí se ha identificado la instancia correspondiente, pero falta dilucidar el momento en que deba hacerse esa calificación y bajo qué criterios.

Respecto de este último punto, es indudable que cualquier calificación de este tipo debe atender a criterios y reglas no sólo nacionales sino internacionales, pues como ya se ha argumentado existe un marco jurídico importante a nivel internacional que protege el derecho de acceso a la información y a la verdad. Y que por ende, ha impuesto obligaciones específicas a los Estados, el no hacerlo acarrearía eventualmente la configuración de la responsabilidad internacional del estado Mexicano. No se debe soslayar que esta determinación de gravedad debe atender a diversos parámetros como ya se ha sostenido y que de ningún modo podría realizarse de manera desinformada, caprichosa o arbitraria.

Sobra decir que la calificación en sí misma es de interés general y por ende, también debe hacerse del dominio público.

Hasta aquí se ha hablado de quién y cómo realizar la clasificación, pero ahora debemos reflexionar respecto del cuándo. En un primer momento, diríamos que se realizará cuando se tengan los elementos necesarios para ello. Pero esta respuesta no ofrece claridad ni certidumbre alguna. Es un momento completamente indeterminado.

Esto es, como se vio, en el propio caso que se comenta aquí, la CNDH no tuvo acceso a la información, pues la Procuraduría se negaba a otorgarla. Esto hace pensar que nos encontramos inmersos en un círculo vicioso: las procuradurías no entregarían información acogiéndose a la excepción referente a que se trata de averiguaciones o indagatorias en curso y sin la información la CNDH se encuentra imposibilitada para calificar los hechos, de ahí que al no haber una calificación, la información de las averiguaciones no se puede publicar.

En este contexto, resulta de fundamental importancia reforzar las facultades de la Comisión en el sentido de que sus peticiones de información sean atendidas insoslayablemente por las procuradurías, fiscalías, juzgados y demás autoridades inmersas en la procuración de justicia; así como garantizar su autonomía a fin de que efectivamente pueda emitir un juicio libre sobre cuándo iniciar una investigación y sobre la calificación de la gravedad. 


\section{Conclusiones}

La Comisión Nacional de Derechos Humanos, de acuerdo al último párrafo del artículo 102 constitucional es la instancia estatal idónea para calificar de graves las violaciones a derechos humanos y con ello lograr que la información relaciona da a los hechos así calificados sea pública sin que pueda la autoridad esgrimir la excepción legal de tratarse de investigaciones vigentes y combatir la opacidad en asuntos trascendentes que lesionan gravemente el orden jurídico y democrático de cualquier país.

Sin embargo, es necesario fortalecer a la CNDH a fin de que realmente sea considerada su autoridad de investigación sobre cualquier órgano estatal, lo que evidentemente también redundará en su autonomía e independencia, cualidades que de no contar con ellas, ninguna comisión protectora de derechos humanos es capaz de cumplir con los altos fines para los que se establece.

Esta sería la forma de asegurar el respeto al derecho de acceso a la información y de instaurar en México un verdadero Derecho a la Verdad, mismo que siempre se ha visto obstaculizado por intereses políticos, partidistas, o de alguna forma hegemónicos.

El artículo $1^{\circ}$ constitucional ha establecido obligaciones a las autoridades que evidentemente deben ser cumplidas y el hecho de garantizar a los gobernados un auténtico acceso a la información redundará en el cumplimiento de las mismas lo que llevará al estado a constituirse en un auténtico Estado constitucional de derechos. Las violaciones que se podrían combatir son del tipo que han llevado a México ante la Corte Interamericana de Derechos Humanos, en donde se ha encontrado responsable.

\section{Bibliografía}

Cancado Trinidade, A. A. (2015). La ampliación del contenido material del contenido material del ius cogens. Washingto, D.C. Recuperado el 31 de Mayo de 2017, de http://www.oas.org/es/ 
sla/ddi/docs/publicaciones_digital_XXXIV_curso_derecho_ internacional_2007_Antonio_Augusto_Cancado_Trindade.pdf. Comisión Interamericana de Derechos Humanos. (1999). LUCIO PARADA CEA, HÉCTOR JOAQUÍN MIRANDA MARROQUÍN, FAUSTO GARCÍA FUNES, ANDRÉS HERNÁNDEZ CARPIO, JOSÉ CATALINO MELÉNDEZ. Washington, D.C.: Organización de Estados Americanos . Obtenido de http://www.cidh.org/ annualrep/98span/Fondo/El\%20Salvador10.480.htm

Comisión Nacional de Derechos Humanos. (23 de Diciembre de 2013). Comisión Nacional de Derechos Humanos. Obtenido de Recomendación No. 80/2013: http://www.cndh.org.mx/sites/all/doc/ Recomendaciones/2013/Rec_2013_080.pdf.

Comité Internacional de la Cruz Roja. (08 de 06 de 1977). Protocolo Adicional II. Obtenido de https://www.icrc.org/spa/resources/ documents/misc/protocolo-i.htm\#6

FUNDAR. (13 de Noviembre de 2015). Centro de Análisis e Información . Obtenido de http://fundar.org.mx/?s=san+fernando\&Search= FUNDAR. (13 de Noviembre de 2015). Solicitan a al corte decidir a favor del derecho a la información y a la verdad en caso de la masacre a migrantes de San Fernando. FUNDAR . Obtenido de http:// fundar.org.mx/solicitan-a-la-corte-decidir-a-favor-del-derecho-a-lainformacion-y-a-la-verdad-en-caso-de-la-masacre-a-migrantes-desan-fernando/?ID=

FUNDAR Centro de Análisis e Investigación. (2015). Reflexiones sobre el Derecho de Acceso a la Información y la iniciativa de Ley General de transparencia. Recuperado el 01 de Junio de 2017, de $\mathrm{http} / / /$ www.senado.gob.mx/comisiones/estudios_legislativos2/docs/ transparencia/Reflexiones_FUNDAR.pdf.

Instituto Federal de Acceso a la Información y Protección de Datos. (2015). Documentos relacionados con los diez puntos que el IFAI entregó al Senado de la República. Obtenido de http://www.senado. gob.mx/comisiones/estudios_legislativos2/docs/transparencia/ IFAI2.PDF. 
Naqvi, Y. (2006). El derecho a la verdad en el derecho internacional: ¿realidad o ficción? International Review of the Red Cross, 33.

Organización de Estados Americanos. (1969). Tratados Multilaterales.

Obtenido de Convención Americana sobre Derechos Humanos: https://www.oas.org/dil/esp/tratados_b-32_convencion_americana_ sobre derechos humanos.htm

Organización de Estados Americanos. (08 de junio de 2010). Ley modelo interamericana sobre acceso a la información pública. Obtenido de http://www.oas.org/es/sla/ddi/acceso_informacion_ley_modelo.asp Organización de las Naciones Unidas. (24 de 03 de 2015). Centro de Información de las Naciones Unidas. Obtenido de http://www.cinu. $\mathrm{mx} /$ comunicados/2015/03/el-derecho-a-la-verdad-es-un-d/

Velásquez Rodríguez vs. Honduras (Corte Interamericana de Derechos Humanos 29 de 07 de 1988). Obtenido de http://www.corteidh.or.cr/ cf/Jurisprudencia2/index.cfm?lang=es

Violaciones graves a derechos humanos. Su concepto para efectos del derecho de acceso ala información de la averiguación previa que las investiga, 2000296 (Primera Sala de la SCJN 2012). Recuperado el 11 de Junio de 2017, de https://sjf.scjn.gob.mx/sjfsist/Paginas/ DetalleGeneralV2.aspx?Epoca $=1 \mathrm{e} 3 \mathrm{e} 10000000000 \&$ Apendice $=10$ $00000000000 \&$ Expresion $=$ violaciones $\% 2520$ graves $\% 2520 \mathrm{a} \% 252$ 0derechos\%2520humanos.\%2520su\%2520concepto\%2520para\%2520 efectos\%2520del\%2520\&Dominio $=$ Rubro,Texto\&TA_TJ $=2 \& O$ 